\title{
Les étudiants de première génération dans les universités : l'accès et la persévérance aux études au Canada ${ }^{1}$
}

Pierre Canisius Kamanzi

Université du Québec à Montréal

Pierre Doray

Université du Québec à Montréal

Sylvie Bonin

Université du Québec

Amélie Groleau

Université du Québec à Montréal

Jake Murdoch

Université de Montréal

\section{RÉSUMÉ}

Les études menées aux États-Unis montrent que les étudiants dits de première génération (ÉPG) sont moins susceptibles de poursuivre leurs études au niveau universitaire, comparativement aux étudiants dont les parents sont titulaires d'un diplôme d'études postsecondaires (collégiales ou universitaires). Les ÉPG qui entreprennent des études universitaires seraient confrontés à plusieurs obstacles qui rendent leur parcours scolaire plus difficile. Qu'en est-il au Canada? Selon les résultats de l'analyse des données de l'Enquête longitudinale auprès des jeunes en transition (EJET), effectuée de 2000 à 2005, la situation est semblable à celles des États-Unis en ce qui concerne l'accès aux études postsecondaires : comparés aux autres étudiants, les ÉPG accèdent à l'université dans une proportion plus faible. Toutefois, on ne constate pas de différence sur le plan de la persévérance : les ÉPG canadiens qui entreprennent des études universitaires obtiennent le diplôme de baccalauréat dans une proportion égale à celle de leurs homologues venant de famille au capital scolaire plus élevé. 


\section{ABSTRACT}

Research in the United States shows that American first generation students (FGS) are less likely to attend university than students whose parents are college graduates (NFGS). Furthermore, those among American FGS who undertake a bachelor degree would have to overcome, throughout their college experience, obstacles related to their family's background. Are the Canadian FGS experiencing the same educational disadvantages than their American counterparts? Using longitudinal data from Youth in Transition Survey (YITS), conducted from 2000 to 2005, this paper shows that, as seen in the American studies, Canadian FGS are less likely to attend university than NFGS. However, there is no difference in regards to academic persistence. FGS and NFGS have the same probability of obtaining a bachelor degree.

\section{INTRODUCTION}

Depuis les années 1960, la démocratisation des études postsecondaires et la lutte contre les inégalités d'accès à l'enseignement postsecondaire sont des visées explicites des politiques éducatives des différents paliers de gouvernements au Canada. Au nom de l'égalité des chances, les gouvernements ont adopté de nombreuses mesures politiques et administratives visant à faciliter l'accès aux études à un plus grand nombre d'étudiants : accroissement des ressources financières allouées aux établissements d'enseignement, création de nouveaux établissements, aide financière aux étudiants démunis, etc. Ces mesures ont permis d'accroître le nombre d'étudiants inscrits au collège et à l'université, si bien qu'au début des années 2000, le Canada a amorcé un virage décisif vers la "massification" de l'enseignement supérieur, pour reprendre l'expression de Dubet (1994). Selon les données de Statistique Canada (2005), le taux d'accès aux études postsecondaires ne cesse d'augmenter. Un récent rapport de l'OCDE (2005) indique que $55,8 \%$ des jeunes Canadiens âgés de 20 à 24 ans détiennent un diplôme d'études postsecondaires ou fréquentent un établissement collégial ou universitaire.

Cette massification s'est traduite par une transformation de la population étudiante en raison de l'augmentation de la proportion d'étudiantes et d'étudiants venant de catégories sociales ou de groupes sociaux auparavant sous-représentés (ex. : femmes, jeunes issus de familles à faible revenu ou de communautés rurales, membres de communautés ethnoculturelles minoritaires et groupes autochtones). Toutefois, en dépit de cette situation, les résultats de recherche soulignent la persistance des inégalités scolaires et donc de l'influence de plusieurs facteurs sociaux et culturels sur l'accessibilité aux études.

À cet égard, des études menées aux États-Unis traitent des parcours scolaires des étudiants dits de première génération (ÉPG, first generation students). Cette notion est née de la nécessité de défınir une catégorie administrative servant à la gestion des programmes d'aide à la réussite dans l'enseignement postsecondaire (Auclair et al., 2008). Par la suite, elle a été utilisée en recherche comme un indicateur du capital scolaire de la famille. Selon les écrits issus pour la plupart de l'Amérique du Nord, et des États-Unis en particulier, deux phénomènes principaux caractérisent les ÉPG : ils ont moins tendance à poursuivre des études universitaires; lorsqu'ils entament de telles études, leur parcours semble plus diffıcile à maints égards. 
Le présent article, qui prend appui sur les données de l'Enquête sur les jeunes en transition (EJET) menée par Statistique Canada et Ressources humaines et Développement des compétences Canada (RHDCC), traite des parcours scolaires des ÉPG qui entreprennent et poursuivent des études universitaires au Canada. Son objectif est d'examiner dans quelle mesure cette notion, essentiellement utilisée aux États-Unis, peut servir à expliquer les inégalités d'accès et de persévérance aux études chez les étudiants canadiens. En même temps, il s'agit de savoir si le statut d'ÉPG constitue un obstacle à l'accès et à la persévérance. L'application de ce concept pour rendre compte de la situation canadienne est justifiée par des raisons liées à la proximité de l'organisation des politiques éducatives et des systèmes d'éducation canadien et étatsunien, et plus globalement, par les points communs de nombreuses politiques sociales entre ces deux pays. En effet, même si, à plusieurs égards, le Canada se rapproche des régimes providentiels socio-démocrates européens, plusieurs études montrent qu'il se classe plutôt parmi les sociétés libérales au côté des États-Unis, du Royaume-Uni et d'autres pays semblables (Saint-Arnaud \& Bernard, 2003; Esping-Andersen, 1999).

Les questions suivantes guident notre démarche :

1. Le statut d'ÉPG est-il un facteur de modulation de l'accès aux études universitaires? Le cas échéant, son influence est-elle directe ou indirecte, agissant seulement par l'intermédiaire d'autres facteurs?

2. Le statut d'ÉPG influe-t-il sur la persévérance aux études, c'est-à-dire le taux d'obtention du diplôme de baccalauréat?

Dans un premier temps, une brève recension des écrits sur la situation des ÉPG aux États-Unis et au Canada est présentée. Compte tenu de l'objet d'étude, seuls les documents qui traitent de la participation et de la persévérance scolaire des ÉPG ont été retenus. De là, les principaux éléments de notre modèle d'analyse ont pu être cernés. Dans un deuxième temps (méthodologie), les données utilisées sont décrites, de même que la manière dont les variables du modèle d'analyse sont défınies et opérationnalisées. Enfin, à la troisième section, les résultats sont présentés et interprétés.

\section{CADRE CONCEPTUEL}

Le concept d'ÉPG a d'abord été utilisé dans le cadre de recherches institutionnelles menées aux États-Unis afin d'examiner l'influence de la scolarité des parents sur les parcours scolaires de leurs enfants (Adachi, 1979; Billson \& Brooks-Terry, 1982). Peu à peu, une tradition de recherche s'est développée autour de ce concept qui a fait l'objet de multiples précisions conceptuelles (Auclair et al., 2008) et théoriques. De façon générale, les études empiriques avaient pour objectif de saisir qui étaient ces étudiants et de quels milieux ils venaient. Elles visaient aussi à comprendre l'influence de la scolarité des parents sur les parcours scolaires de leurs enfants.

Dans son usage administratif, la catégorie "ÉPG " est relativement large. Pour les programmes fédéraux étasuniens TRIO par exemple - programmes qui financent des interventions en matière d'égalité d'accès aux études - un ÉPG est un étudiant dont les parents n'ont pas obtenu de diplôme de baccalauréat (college degree). Cette définition inclut donc les étudiants dont les parents ont eu une expérience postsecondaire, sans nécessairement avoir atteint le grade de bachelier. Toutefois, peu de chercheurs emploient cette définition dans le cadre de leurs analyses (Dennis, Phinney \& Chateco, 2005; Pike \& Kuh, 2005; Ishitani, 2003; Naumann, Bandalos \& Gutkin, 2003; Penrose, 2002). 
Dans la plupart des travaux scientifiques, un ÉPG est un étudiant issu d'une famille où les parents n'ont jamais fréquenté un établissement postsecondaire collégial ou universitaire. En effet, la majorité des chercheurs estime que le fait d'avoir fréquenté un collège ou une université est suffisant pour qu'un individu ait des connaissances sur l'éducation postsecondaire et possède un capital social et culturel transmissible à ses enfants lors de la transition vers ce niveau d'études (Lohfınk \& Paulsen, 2005; Pascarella et al., 2004, 2003). Toutefois, la définition des étudiants dits de seconde génération varie d'une recherche à l'autre (Auclair et al., 2008).

Compte tenu des données dont nous disposons, la définition retenue est basée sur le diplôme le plus élevé de l'un des parents et se décline en trois catégories : 1) les ÉPG sont les étudiants dont aucun parent n'est titulaire d'un diplôme d'études postsecondaires, 2) les NÉPG collégiaux sont ceux dont le parent le plus scolarisé détient un diplôme d'études collégiales et 3) les NÉPG universitaires sont ceux dont au moins un parent est titulaire d'un diplôme universitaire.

\section{Les ÉPG et l'accès aux études postsecondaires aux États-Unis}

Dans le corpus des travaux, l'accès aux études postsecondaires est abordé sous l'angle de la répartition des différents groupes sociaux au sein des établissements postsecondaires. Dans cette optique, les études qui traitent de l'accès des ÉPG aux études supérieures tendent à montrer des différences en comparaison avec leurs pairs par rapport à certaines caractéristiques démographiques et sociales. Par exemple, les travaux de Horn et Nuñez (2000) indiquent que les ÉPG étasuniens viennent davantage des familles à faible revenu, des communautés hispaniques et noires ou des familles monoparentales. Par ailleurs, on constate que les ÉPG qui entrent au collège ou à l'université sont généralement plus âgés que les NÉPG (Warburton, Bugarin \& Nuñez, 2001; Inman \& Mayes, 1999; Brown \& Burkhardt, 1998; Nuñez, Cuccaro-Alamin \& Caroll, 1998).

Ces différences de composition de l'origine culturelle et sociale conduisent à s'interroger sur l'influence respective des différents "facteurs" sur les parcours scolaires. Les ÉPG sont-ils moins nombreux à poursuivre des études universitaires parce que la composition du capital scolaire de la famille influence leur choix ou parce que les autres modes d'ancrage social modulent leur décision?

Comme le notent Horn et Nuñez (2000), un des obstacles les plus importants à la participation aux études postsecondaires pour les ÉPG serait le manque d'expérience des parents dans le processus de transition entre le secondaire et le postsecondaire. En effet, pour les parents des ÉPG, il est impossible de transmettre le bagage culturel lié aux études postsecondaires puisqu'ils n'ont eux-mêmes pas eu la chance de l'acquérir. Les ÉPG ne souffriraient donc pas d'un déficit intrinsèque et incontournable, mais plutôt d'un manque d'information et de modèles ce qui, par conséquent, réduirait leur accès aux études postsecondaires (Horn \& Nuñez, 2000). York-Anderson et Bowman (1991) rapportent des différences importantes en ce qui concerne les connaissances de base des ÉPG sur le postsecondaire, leur engagement personnel dans les études et enfin, le soutien qu'ils reçoivent de leurs familles.

Les ÉPG seraient aussi nettement désavantagés par leur préparation scolaire avant d'entrer à l'université. Ils recevraient moins de soutien à l'école et à la maison et auraient davantage de difficultés d'adaptation et d'intégration sociale et scolaire (Horn 
\& Nuñez, 2000). Parmi ceux qui entreprennent des études postsecondaires, plusieurs vivraient des situations de stress et d'anxiété qui rendent leur expérience scolaire plutôt difficile (Lara, 1992; Rendon, 1992; Rodriguez, 1982, 1975).

En résumé, comparativement à leurs pairs dont les parents ont fréquenté le postsecondaire, les ÉPG sont moins susceptibles de participer aux études supérieures. Même si la qualité de la préparation scolaire et le soutien de la famille constituent des facteurs indiscutables d'accès et de persévérance aux études universitaires, ils n'expliquent qu'en partie les différences observées entre les ÉPG et les autres étudiants. En plus de s'impliquer dans la scolarité de leurs enfants, les parents des NÉPG leur lèguent un modèle social et culturel qui favorise le développement d'aspirations universitaires.

\section{Les ÉPG et la poursuite des études postsecondaires aux États-Unis}

Les travaux portant sur la persévérance aux études chez les ÉPG abordent également l'expérience scolaire dans les collèges ou les universités selon différents aspects, dont la persévérance aux études et la réussite scolaire. Leurs résultats montrent que le fait d'être issu d'une famille faiblement scolarisée constitue un obstacle à la persévérance scolaire (Billson \& Brooks-Terry, 1982; Nuñez, Cuccaro-Alamin \& Carroll, 1998; Choy, 2001, Warburton, Bugarin \& Nuñez, 2001; Ishitani, 2003, 2006; Lohfink \& Paulsen, 2005). Toutefois, tous les travaux n'aboutissent pas à cette conclusion. Ainsi, Chen et Carroll (2005), à partir d'une analyse multivariée fondée sur un échantillon national de finissants de l'école secondaire aux États-Unis, n'ont pas remarqué de différence significative de persévérance entre les ÉPG et les autres étudiants, tant en ce qui a trait à la poursuite d'études postsecondaires en général, que pour la fréquentation d'un collège de quatre ans (Four-Year College) (Chen \& Carroll, 2005 : 49). Ils notent que les ÉPG sont moins susceptibles d'obtenir un diplôme de premier cycle universitaire (Chen \& Carroll, 1995 : 53) et sont beaucoup plus nombreux à suivre des cours de rattrapage.

À l'instar de l'accès aux études postsecondaires, la persévérance peut être modulée par les caractéristiques sociodémographiques de l'individu, comme l'observent Lohfink et Paulsen (2005). Elle serait, entre autres, influencée par la classe sociale, le sexe et l'appartenance ethnoculturelle. Par ailleurs, Pascarella, Wolniak, Pierson et Terenzini (2004) ont aussi constaté que les ÉPG qui entraient à l'université sont fortement désavantagés par rapport à leur expérience scolaire et leur vie extrascolaire. Ils ont moins tendance à vivre sur le campus que les autres étudiants, à s'engager dans les activités parascolaires et scolaires et à s'intégrer à une fraternité étudiante. Or, les analyses montrent que ces activités ont des effets positifs sur le développement de la pensée critique, les chances d'obtenir le diplôme et la préférence pour des tâches intellectuelles de haut niveau.

Des différences se font aussi sentir sur d'autres aspects de la fréquentation scolaire : le temps alloué aux études, le type d'établissement choisi, le nombre d'heures de cours et d'heures au travail ainsi que le type de cours et de programmes sélectionnés. Pascarella, Wolniak, Pierson, et Terenzini (2003) ont montré que les ÉPG consacrent moins de temps à leurs études. Dans le même sens, Nuñez, Cuccaro-Alamin et Carroll (1998) ainsi que Warburton, Bugarin et Nuñez (2001) ont constaté que les ÉPG sont plus susceptibles de s'inscrire aux études à temps partiel et de travailler à temps plein, alors que leurs pairs NÉPG sont plus nombreux à adopter un mode "régulier " de fréquentation des études. 
Les ÉPG qui entreprennent des études postsecondaires seraient également confrontés à des difficultés d'adaptation scolaire. Depuis la publication de l'article de Billson et Brooks-Terry (1982) sur l'intégration sociale et intellectuelle des ÉPG et son impact sur la persévérance au collège, plusieurs chercheurs se sont intéressés à cette dimension de l'expérience scolaire.

Dans une analyse qualitative des transformations culturelles lors du passage vers l'enseignement postsecondaire, London $(1989,1996)$ décrit ce passage comme un moment de séparation avec la famille. Plusieurs ÉPG, n'ayant pas grandi avec l'idée de fréquenter l'enseignement postsecondaire, vivent un processus de profonde transformation au moment de leur entrée. La phase de transition aux études postsecondaires est davantage marquée par des difficultés d'adaptation scolaire et d'appropriation de la culture institutionnelle (Hahs-Vaughn, 2004; Terenzini, Springer, Yaeger, Pascarella \& Nora, 1996). Mais l'étude de Pascarella, Wolniak, Pierson et Terenzini (2004), réalisée auprès d'étudiants de collèges de quatre ans, montre que l'expérience scolaire des ÉPG se différencie de celle des autres étudiants, mais qu'après l'obtention d'un diplôme universitaire, il n'y pas de différence quant l'accès à l'emploi et au type d'occupation. L'écart semble diminuer, voire disparaître, avec le temps (Ishitani, 2006; Hahs-Vaughn, 2004; Duggan, 2002).

Par ailleurs, Pike et Kuh (2005) ont constaté que les ÉPG sont moins engagés dans la vie institutionnelle que les autres étudiants, perçoivent le contexte institutionnel comme moins soutenant et ressentent une plus faible progression de leur développement intellectuel et de leurs apprentissages. Toutefois, ils précisent que ce niveau plus faible d'engagement, résultat indirect du statut d'ÉPG, est davantage lié aux aspirations scolaires plus faibles, ainsi qu'au fait de vivre hors du campus.

\section{Le capital scolaire des parents et l'enseignement postsecondaire canadien}

Au Canada comme aux États-Unis, l'impact de la scolarité des parents sur l'accès aux études postsecondaires a déjà été partiellement établi par Rahman, Situ et Jimmo (2005). Ces auteurs ont montré que cette variable agit indépendamment de la provenance régionale ou rurale, du sexe, de la structure et du revenu familial. D'autres études fondées sur les données de l'EJET ont corroboré l'existence de cette relation positive entre le niveau de scolarité des parents et la participation des jeunes Canadiens aux études postsecondaires. Elles ont également révélé que cette relation est indépendante des autres variables connues pour leur influence sur l'accès et la persévérance aux études, telles que l'âge, le système scolaire ou le type de famille (Finnie, Laporte \& Lascelles, 2004; Shaienks \& Gluszynski, 2007; Finnie, Lascelles \& Sweetman, 2005).

Au Canada, le concept d'ÉPG a suscité l'intérêt des chercheurs, mais son usage demeure à ce jour très limité. Les travaux canadiens qui l'utilisent de manière explicite pour rendre compte de l'expérience scolaire des parents sont encore peu nombreux. Par exemple, au sujet de l'accès aux études, Kamanzi et al. (2009) l'ont utilisé pour cerner l'influence du capital scolaire des parents sur l'accès aux études postsecondaires. Les résultats de l'analyse des données longitudinales de l'EJET recueillies auprès d'un panel de jeunes âgés de 15 ans en 1999, et de 21 ans en 2005, indiquent que même lorsque l'on tient compte des variables sociodémographiques (sexe, catégorie socioprofessionnelle des parents, etc.) et des antécédents scolaires, l'influence de la scolarité des parents sur 
l'accès aux études postsecondaires se maintient. Les ÉPG sont moins susceptibles que leurs pairs d'accéder aux études collégiales, et encore moins à l'université. Cependant, les données utilisées dans cette recherche ne permettaient pas d'aborder la question de persévérance puisque la durée d'observation du séjour à l'université était encore trop courte pour que la majorité des répondants aient pu obtenir un diplôme.

Grayson (1997) a lui aussi abordé ce concept pour étudier les relations entre les caractéristiques sociodémographiques des étudiants, le niveau de scolarité des parents, le genre, le revenu familial et les résultats scolaires au secondaire, les expériences institutionnelles et les moyennes cumulatives. Il a constaté que les ÉPG inscrits à l'Université York de Toronto avaient une moyenne cumulative plus faible que les autres étudiants et étaient moins engagés dans des activités extrascolaires. Toutefois, il n'y avait pas de différence concernant leur engagement scolaire. Dans une perspective semblable, Lehmann (2007) a appliqué le concept d'ÉPG à l'analyse de l'expérience scolaire des ÉPG dans une université du sud-ouest de l'Ontario, afın de comprendre le lien entre le statut d'ÉPG, la classe sociale et l'abandon scolaire. Ses résultats révèlent que les ÉPG sont plus susceptibles d'abandonner les études de manière prématurée, même lorsqu'ils ont de bons résultats scolaires.

La présente étude n'est donc pas la première à aborder l'effet du statut d'ÉPG dans le contexte des études postsecondaires au Canada. Toutefois, les rares études sur le sujet ne traitent pas de la persévérance scolaire qui, aux yeux des analystes et des décideurs, comporte une dimension économique et sociale importante à plusieurs égards. Afın de mieux comprendre la situation canadienne, le présent article vise donc à examiner, à partir de données empiriques canadiennes, si le statut d'ÉPG exerce une influence sur l'accès et la persévérance aux études universitaires. Plus précisément, la question consiste d'abord à savoir si, au Canada, l'influence de cette variable se limite à l'accès aux études postsecondaires, se maintient au cours de la fréquentation des études postsecondaires ou disparaît. L'article vise également à examiner si le fait d'être ÉPG influe sur d'autres aspects du parcours scolaire, entre autres l'obtention d'un diplôme de baccalauréat. Pour ce faire, nous avons choisi d'utiliser des données longitudinales obtenues auprès des jeunes adultes de 24 à 26 ans.

\section{MÉTHODOLOGIE}

\section{Source des données}

L'étude s'appuie sur les données pancanadiennes de l'EJET (cohorte B) menée par RHDCC et Statistique Canada depuis 2000. Cette enquête longitudinale (quatre cycles) porte sur un panel de jeunes Canadiens âgés de 18 à 20 ans en 1999, puis de 24 à 26 ans en 2005. Au total, le sous-échantillon utilisé est composé de 10882 sujets issus de l'ensemble des provinces canadiennes et ayant répondu au questionnaire de l'enquête jusqu'au cycle 4 (2005).

Compte tenu de la problématique étudiée (l'influence du statut d'ÉPG sur l'accès et la persévérance aux études universitaires), nous utiliserons les données des quatre cycles de l'enquête. La base de données contient des renseignements sur le niveau de scolarité des parents des répondants. En outre, des données ont été compilées sur le parcours scolaire des étudiants tant au secondaire qu'au postsecondaire : les résultats scolaires au secondaire, certaines variables caractérisant le niveau d'engagement aux études 
secondaires et postsecondaires (le temps alloué aux devoirs et le décrochage scolaire), le type d'établissement scolaire fréquenté, le domaine d'études, le régime d'études, etc. L'EJET a aussi permis de recueillir un ensemble de données sur les caractéristiques sociodémographiques des élèves (sexe, groupe d'appartenance ethnolinguistique, pays de naissance, âge et année d'entrée à l'université) et l'environnement dans lequel ils ont évolué. Bref, la base de données permet de dresser un portrait général des jeunes qui fréquentent un établissement universitaire.

\section{Opérationnalisation des variables}

Deux variables dépendantes sont étudiées : l'accès aux études universitaires et l'obtention du diplôme de baccalauréat. Plusieurs variables indépendantes sont intégrées au modèle. Le statut d'ÉPG constitue la variable indépendante principale. Nous avons tenu compte de quatre autres groupes de variables : l'appartenance sociale et culturelle, les antécédents scolaires au secondaire, les modes de cheminement scolaire et l'offre des études postsecondaires.

Les variables d'appartenance sociale et culturelle incluent le capital scolaire des parents (ÉPG, NÉPG collégial et NÉPG universitaire), l'appartenance linguistique, l'appartenance à une minorité visible et le milieu de résidence (région rurale ou urbaine). Les antécédents scolaires font référence à la scolarité au secondaire: moyenne générale, temps consacré aux devoirs, épisode de décrochage, interruption d'études avant l'entrée à l'université, type d'établissement secondaire fréquenté (privé ou public) et aspirations scolaires. Les variables de cheminement scolaire sont : l'âge d'entrée à l'université, le domaine d'études et le régime d'études.

Dans la mesure où l'éducation est de responsabilité provinciale, il est possible que l'offre des études universitaires varie d'une province à l'autre. Pour tenir compte de ces différences, deux variables sont incluses dans le modèle d'analyse : la province et le type d'université fréquentée. La variable "province " permet de tenir compte des politiques éducatives spécifiques de chaque province, mais aussi de leurs caractéristiques socioéconomiques respectives. Pour le type d'université, nous distinguons les anciennes universités (fondées en 1959 ou avant) et les nouvelles universités (fondées après 1959).

Nous proposons une analyse descriptive et une analyse multivariée. La première vise à déterminer, à l'aide du test khi-carré, les variables indépendantes exerçant une influence significative sur la participation aux études universitaires et la chance d'obtenir un diplôme de baccalauréat, en mettant l'accent sur le statut d'ÉPG. Elle a aussi pour objectif de dresser le portrait général des étudiants persévérants en fonction de leurs caractéristiques sociodémographiques, de leur expérience scolaire et de leur cheminement universitaire.

L'analyse multivariée permet d'estimer l'influence relative de chacune des variables de l'appartenance sociale et culturelle et de la scolarité antérieure sur la probabilité d'accéder à l'université, d'obtenir un diplôme de baccalauréat ou de poursuivre des études supérieures, l'effet des autres variables étant contrôlé. La régression logistique a été privilégiée. Dans la première équation (modèle 1), seule la variable ÉPG a été examinée, alors que dans la seconde (modèle 2), des variables associées à l'appartenance sociale et culturelle et à la scolarité ont été ajoutées. 


\section{RÉSULTATS}

\section{L'accès aux études universitaires}

La plupart des jeunes Canadiens poursuivent des études postsecondaires. Dans le cadre de la présente étude, environ $34 \%$ des répondants étaient inscrits dans un collège et $47 \%$ dans une université. Tandis que le passage aux études postsecondaires se veut fortement modulé par la scolarité des parents, les ÉPG seraient désavantagés en ce qui concerne l'accès à l'université. Comme l'indique la figure 1, le taux de participation est nettement supérieur chez les NÉPG, en comparaison aux ÉPG. Il est respectivement de $69 \%$ et de $44 \%$ pour les NÉPG universitaires et les NÉPG collégiaux, alors qu'il est de $20 \%$ pour les ÉPG (voir figure 1).

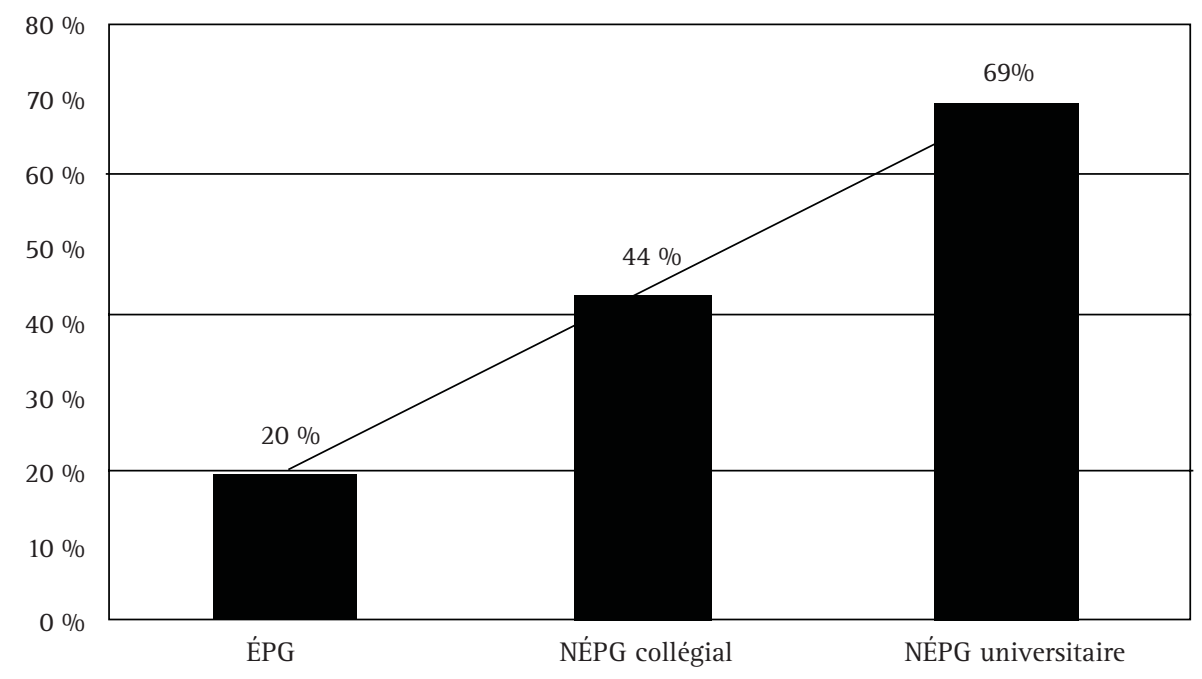

Figure 1

Taux d'accès aux études universitaires selon le statut d'ÉPG

Les résultats des régressions logistiques (tableau 1) indiquent que l'accès aux études universitaires s'accroît avec la scolarité des parents : les ÉPG sont nettement moins susceptibles d'accéder aux études universitaires (modèle 1). Cette relation demeure significative même lorsque d'autres facteurs liés à l'origine sociale, à la scolarité et à l'offre des études sont pris en compte (modèle 2). La probabilité de fréquenter une université augmente respectivement de 1,68 fois et de 3,29 fois pour les NÉPG collégiaux et les NÉPG universitaires.

Parmi les facteurs d'appartenance sociale et culturelle, seuls le type d'occupation $\mathrm{du}$ père et le lieu de résidence influencent de façon significative l'accès aux études universitaires lorsque les autres variables sont incluses dans le modèle d'analyse. Avoir un père occupant un poste de cadre supérieur ou de professionnel accroît la probabilité d'accès aux études universitaires respectivement de $66 \%(1,66$ - 1$)$ et de $74 \%(1,74-1)$ en comparaison aux postes techniques et para-professionnels (catégorie de référence). Habiter en région rurale réduit cette même probabilité de $46 \%(0,54-1)$. 
Tableau 1

Influence des caractéristiques d'appartenance sociale et culturelle et du parcours scolaire sur l'accès aux études universitaires au Canada - Rapports des cotes de régression logistique

\begin{tabular}{|c|c|c|}
\hline & \multicolumn{2}{|c|}{ Rapports des cotes } \\
\hline & Modèle 1 & Modèle 2 \\
\hline \multicolumn{3}{|l|}{ Étudiants de première génération } \\
\hline ÉPG & & Réf. \\
\hline NÉPG collégial & $1,95^{* * *}$ & $1,68^{* * * *}$ \\
\hline NÉPG universitaire & $5,60^{* * * *}$ & $3,29^{* * * *}$ \\
\hline \multicolumn{3}{|l|}{ Sexe } \\
\hline Femme & - & 1,21 \\
\hline Homme & & Réf. \\
\hline \multicolumn{3}{|l|}{ Emploi du père } \\
\hline Cadre supérieur et directeur & - & $1,66^{* * * *}$ \\
\hline Poste professionnel & - & $1,74^{* * *}$ \\
\hline Postes technique et paraprofessionnel & - & Réf. \\
\hline Poste intermédiaire & - & 0,94 \\
\hline Poste élémentaire & - & $1,22^{* *}$ \\
\hline Sans emploi ou retraité & - & 0,54 \\
\hline \multicolumn{3}{|l|}{ Emploi de la mère } \\
\hline Cadre supérieure et directrice & - & 1,29 \\
\hline Poste professionnel & - & 1,17 \\
\hline Postes technique et paraprofessionnel & - & Réf. \\
\hline Poste intermédiaire & - & 0,89 \\
\hline Poste élémentaire & - & 0,77 \\
\hline Sans emploi ou retraitée & - & 1,22 \\
\hline \multicolumn{3}{|l|}{ Appartenance linguistique } \\
\hline Anglophone du Québec & - & 0,95 \\
\hline Anglophone des autres provinces & - & Ref. \\
\hline Francophone hors Québec & - & 1,02 \\
\hline Francophone du Québec & - & 1,01 \\
\hline Allophone & - & 1,16 \\
\hline \multicolumn{3}{|l|}{ Appartenance à une minorité visible } \\
\hline Oui & - & $1,73^{*}$ \\
\hline non & - & Réf. \\
\hline \multicolumn{3}{|l|}{ Lieu de résidence } \\
\hline Région rurale & - & $0,54^{* * *}$ \\
\hline Région urbaine & - & Réf. \\
\hline \multicolumn{3}{|l|}{ Moyenne générale au secondaire } \\
\hline $90-100 \%$ & - & $24,90^{* * * *}$ \\
\hline $80-89 \%$ & - & $8,69^{* * * *}$ \\
\hline $70-79 \%$ & - & $3,03^{* * * *}$ \\
\hline Moins de $70 \%$ & - & Réf. \\
\hline \multicolumn{3}{|l|}{ Temps consacré aux devoirs par semaine } \\
\hline 8 heures ou plus & - & $2,70^{* * * *}$ \\
\hline 4 à 7 heures & - & $2,11^{* * *}$ \\
\hline 1 à 3 heure(s) & - & $1,41^{*}$ \\
\hline Moins d'une heure & - & Réf. \\
\hline \multicolumn{3}{|l|}{ Interruption temporaire d'études } \\
\hline Oui & - & $0,21^{* * *}$ \\
\hline Non & - & Réf. \\
\hline \multicolumn{3}{|l|}{ Type d'établissement secondaire fréquenté } \\
\hline Privé & - & 1,36 \\
\hline Public & - & Réf. \\
\hline
\end{tabular}




\begin{tabular}{|c|c|c|c|}
\hline & & \multicolumn{2}{|c|}{ Rapports des cotes } \\
\hline & & Modèle 1 & Modèle 2 \\
\hline \multicolumn{4}{|l|}{ Province de résidence } \\
\hline Terre-Neuve-et-Labrador & & - & $2,07^{* * * *}$ \\
\hline Île-du-Prince-Édouard & & - & $2,06^{* * * *}$ \\
\hline Nouvelle-Écosse & & - & $2,01^{* * * *}$ \\
\hline Nouveau-Brunswick & & - & $1,59^{* * *}$ \\
\hline Québec & & - & 0,84 \\
\hline Ontario & & - & Réf. \\
\hline Manitoba & & - & $1,81^{* * * *}$ \\
\hline Saskatchewan & & - & $1,83^{* * * *}$ \\
\hline Alberta & & - & 1,22 \\
\hline \multirow[t]{5}{*}{ Colombie-Britannique } & & - & 0,93 \\
\hline & Pseudo- $\mathrm{R}^{2 \mathrm{~s}}$ & 0,09 & 0,28 \\
\hline & $\chi_{\text {de Wald }}^{2}$ & $480,84^{* * * *}$ & $904,30^{* * * *}$ \\
\hline & & 2 & 36 \\
\hline & $\mathrm{N}$ & 10784 & 7887 \\
\hline
\end{tabular}

${ }^{*} \mathrm{p}<0,05 ;{ }^{* * *} \mathrm{p}<0,01 ; * * * \mathrm{p}<0,001$

L'accès est aussi modulé par la province de résidence. Ainsi, le fait d'habiter les provinces atlantiques, le Manitoba et la Saskatchewan accroît la probabilité d'accéder aux études universitaires en comparaison avec l'Ontario (catégorie de référence). Sur le plan de la scolarité antérieure, la moyenne générale au secondaire et le type d'établissement secondaire fréquenté exercent une influence significative sur les chances de poursuivre les études universitaires. Les rapports de cotes montrent que les élèves ayant une moyenne de 90 à $100 \%$ ont huit fois plus de chances d'étudier à l'université que ceux dont la moyenne est de $70 \%$ ou moins (catégorie de référence). Du coup, on peut aussi en déduire que les premiers sont également presque trois fois $(24,90 / 8,69)$ plus susceptibles de fréquenter une université que ceux ayant une moyenne de 80 à $90 \%$. Dans le même ordre d'idées, les élèves qui consacrent huit heures ou plus par semaine aux devoirs sont 2,70 fois plus susceptibles de poursuivre les études universitaires que ceux qui y consacrent moins d'une heure. Notons également qu'ils ont également presque deux fois plus de chances de participer aux études universitaires que ceux qui accordent seulement une à trois heures par semaine aux devoirs.

En résumé, l'accès aux études universitaires dépend de plusieurs dimensions. La première est la nature de l'offre des services éducatifs dans chacune des provinces. À cet égard, l'importance que prennent les universités dans l'ensemble des établissements d'enseignement postsecondaire dans une région donnée est un facteur à considérer. C'est ce qui explique les différences statistiquement significatives entre les provinces. Ainsi, les provinces qui se distinguent significativement de l'Ontario seraient celles où les universités ont un poids relatif plus élevé au sein de l'enseignement postsecondaire parce que l'enseignement collégial y est moins développé. Dans ces conditions, les jeunes qui veulent poursuivre des études postsecondaires vont se diriger davantage vers l'université. L'influence du lieu de résidence (habiter en milieu rural en comparaison au milieu urbain) pourrait s'expliquer par l'éloignement de l'établissement universitaire le plus proche par rapport au lieu d'habitation familiale (Frenette, 2002, 2003).

La deuxième dimension, de nature individuelle, a trait au niveau de capital scolaire de la famille et à l'occupation du père. Ces deux variables ont une influence qui perdure même quand des caractéristiques de la scolarité au secondaire sont contrôlées. Nous 
retrouvons largement la tendance à la reproduction sociale. La décision de poursuivre les études universitaires est influencée par le soutien familial et la socialisation primaire en général qui relèvent du statut socioéconomique des parents et donc des différents types de ressources dont ils disposent.

\section{La persévérance jusqu'à l'obtention du diplôme de baccalauréat}

Dans quelle mesure la persévérance jusqu'à l'obtention du diplôme est-elle influencée par la scolarité des parents? Pour répondre à cette question, nous avons choisi d'examiner le cas des 4454 étudiants qui, au premier programme, s'étaient inscrits au baccalauréat. Parmi eux, certains étaient sortis du système scolaire avec ou sans diplôme, d'autres poursuivent encore des études au premier cycle ou aux études supérieures.

Les résultats de l'analyse descriptive (tableau 2) montrent qu'au cycle 4, pour l'ensemble des répondants, $56 \%$ des étudiants avaient obtenu leur diplôme de baccalauréat, alors que $44 \%$ étaient encore aux études ou les avaient quittées. Les chances d'obtention du diplôme sont relativement égales pour tous les étudiants, quelle que soit la scolarité des parents. Les résultats indiquent qu'au cycle 4 de l'enquête, $53 \%$ des ÉPG et des NÉPG collégiaux avaient obtenu le diplôme de baccalauréat, contre 59 \% des NÉPG universitaires. Le fait d'être ÉPG n’influe pas de manière significative sur la probabilité d'obtention du diplôme.

En ce qui a trait aux caractéristiques d'appartenance sociale, l'obtention du diplôme est associée à l'occupation du père et au sexe de l'étudiant. Les étudiants dont les pères occupent des postes de cadre ou de professionnel obtiennent le diplôme de baccalauréat en plus grande proportion que les autres. Le taux d'obtention du diplôme est aussi plus élevé chez les femmes (60\%) que chez les hommes (52\%).

Le taux d'obtention du diplôme est également plus élevé chez les étudiants âgés de 26 ans que chez les plus jeunes, mais cette différence n'est pas significative. C'est surtout l'année d'entrée aux études qui fait la différence : le taux d'obtention du diplôme varie de 61 à $63 \%$ chez ceux qui ont commencé des études universitaires entre 1997 et 2000, alors qu'il est de $37 \%$ chez ceux qui ont commencé après 2000. Par rapport à la province, le taux d'obtention du diplôme est un peu plus élevé au Québec (61\%) et en Ontario (59\%), alors qu'il est plus faible à Terre-Neuve-et-Labrador (40\%) ainsi qu'à l'Île-du-Prince-Édouard (46\%). Il n’y a pas de différence significative selon le milieu de résidence de l'étudiant (rural/urbain), ni selon l'origine ethnique ou linguistique.

Enfin, les chances d'obtenir un diplôme de baccalauréat sont associées à la qualité de la scolarité de l'étudiant au secondaire. L'obtention du diplôme est liée aux compétences scolaires antérieures et au niveau d'engagement aux études. Le tableau 3 indique que ces chances sont plus élevées chez les étudiants qui, au secondaire, avaient des moyennes générales élevées, consacraient beaucoup plus de temps aux devoirs et n'avaient pas connu d'irrégularité comme le décrochage ou l'interruption des études après le secondaire. Ainsi, la proportion des répondants qui avaient déjà obtenu un diplôme de baccalauréat au cycle 4 de l'enquête est de $70 \%$ lorsque leur moyenne générale du secondaire se situe entre 90 et $100 \%$, alors qu'elle est de $20 \%$ chez ceux dont la moyenne générale est inférieure à $70 \%$. Elle est de $63 \%$ chez les répondants qui affirmaient consacrer huit heures ou plus aux devoirs par semaine au secondaire, alors qu'elle est de $28 \%$ chez ceux qui déclaraient y accorder moins d'une 
Tableau 2

Pourcentage d'obtention du diplôme de baccalauréat selon le niveau de scolarité des parents et les caractéristiques d'appartenance sociale et culturelle

Statut d'ÉPG $\left(\chi^{2}(2)=11,33, \mathrm{NS}\right)$

ÉPG

Sans diplôme

Diplôme

NÉPG collégial

NÉPG universitaire

Occupation du père $\left(\chi^{2}(5)=36,00 ; p<0,01\right)$

Cadre supérieur ou directeur

Poste professionnel

Poste technique ou paraprofessionnel 53

Poste intermédiaire 56

Poste élémentaire et de manœuvre

Sans emploi ou retraité

Occupation de la mère $\left(\chi^{2}(5)=7,78\right.$; NS)

Cadre supérieure ou directrice

Poste professionnel

Poste technique ou paraprofessionnel 57

Poste intermédiaire 57

Poste élémentaire et de manœuvre 48

Sans emploi ou retraitée

Sexe $\left(\chi^{2}(1)=24,11 ; p<0,01\right)$

Homme

Femme

Âge en $2005\left(\chi^{2}(2)=15,90 ; N S\right)$

24 ans

25 ans

26 ans

Année de début des études universitaires $\left(\chi^{2}(4)=238,13 ; p<0,001\right)$

1997 ou avant 63

1998

1999 61

2000 61

2001 ou après

Minorité visible $\left(\chi^{2}(1)=3,58 ; N S\right)$

Non

Oui

Origine ethnolinguistique $\left(\chi^{2}(4)=17,53\right.$; NS)

Anglophone hors Québec

Francophone hors Québec

Francophone du Québec

61

Anglophone du Québec

Allophone

Lieu de résidence $\left(\chi^{2}(1)=0,48\right.$; NS)

Région rurale

Région urbaine 
Province de résidence $\left(\chi^{2}(9)=47,56 ; p<0,01\right.$

\begin{tabular}{lll} 
Terre-Neuve-et-Labrador & 60 & 40 \\
Île-du-Prince-Édouard & 54 & 46 \\
Nouvelle-Écosse & 42 & 58 \\
Nouveau-Brunswick & 45 & 55 \\
Québec & 39 & 61 \\
Ontario & 41 & 59 \\
Manitoba & 53 & 47 \\
Saskatchewan & 53 & 48 \\
Alberta & 52 & 48 \\
Colombie-Britannique & 52 & 48 \\
\multicolumn{1}{c}{ Ensemble des répondants } & 44 & 56
\end{tabular}

NS: non significatif au seuil de 0,05.

heure par semaine. Les personnes ayant vécu une expérience de décrochage scolaire ou ayant interrompu les études avant le passage à l'université ont moins de chances (respectivement $11 \%$ et $18 \%$ ) d'obtenir le diplôme de baccalauréat.

\section{Tableau 3}

Pourcentage lié à l'obtention du diplôme de baccalauréat selon les caractéristiques de la scolarité au secondaire

\begin{tabular}{|c|c|c|}
\hline & $\begin{array}{c}\text { Sans } \\
\text { diplôme }\end{array}$ & Diplôme \\
\hline \multicolumn{3}{|l|}{ Moyenne générale $\left(\chi^{2}(3)=234,38 ; p<0,001\right)$} \\
\hline $90-100 \%$ & 30 & 70 \\
\hline $80-90 \%$ & 38 & 62 \\
\hline $70-80 \%$ & 55 & 45 \\
\hline Moins de $70 \%$ & 80 & 20 \\
\hline \multicolumn{3}{|l|}{ Avoir connu un décrochage scolaire $\left(\chi^{2}(1)=79,46 ; p<0,001\right)$} \\
\hline Non & 43 & 57 \\
\hline Oui & 89 & 11 \\
\hline \multicolumn{3}{|l|}{ Avoir temporairement interrompu les études $\left(\chi^{2}(1)=169,84 ; p<0,001\right)$} \\
\hline Non & 41 & 59 \\
\hline Oui & 82 & 18 \\
\hline \multicolumn{3}{|l|}{$\begin{array}{l}\text { Temps accordé aux devoirs par semaine } \\
\left(\chi^{2}(3)=100,73 ; p<0,001\right)\end{array}$} \\
\hline Moins de 1 heure & 72 & 28 \\
\hline 1 - 3 heure(s) & 51 & 49 \\
\hline $4-7$ heures & 46 & 54 \\
\hline 8 heures ou plus & 37 & 63 \\
\hline \multicolumn{3}{|l|}{ Obtention du diplôme d'études secondaires avant 18 ans $\left(\chi^{2}(1)=1,20\right.$; NS) } \\
\hline Oui & 42 & 58 \\
\hline Non & 44 & 56 \\
\hline \multicolumn{3}{|l|}{$\begin{array}{l}\text { Niveau d'aspirations scolaires } \\
\left(\chi^{2}(2)=286,50 ; p<0,001\right)\end{array}$} \\
\hline Études collégiales & 83 & 17 \\
\hline Études de premier cycle universitaire & 44 & 56 \\
\hline Études de deuxième cycle universitaire & 35 & 65 \\
\hline \multicolumn{3}{|l|}{ Type d'établissement secondaire fréquenté $\left(\chi^{2}(1)=0,35 ; \mathrm{NS}\right)$} \\
\hline Privé & 44 & 56 \\
\hline Public & 46 & 54 \\
\hline Ensemble des répondants & 44 & 56 \\
\hline
\end{tabular}

NS: non significatif au seuil de 0,05. 
Le taux d'obtention du diplôme est plus élevé chez les étudiants dont le niveau d'aspiration scolaire est élevé dès le secondaire. Il est de $65 \%$ chez ceux qui, avant l'entrée à l'université, aspiraient déjà à un diplôme d'études universitaires de deuxième cycle, alors qu'il est de $17 \%$ chez ceux qui aspiraient à un diplôme d'études collégiales.

Les chances d'obtenir un diplôme dépendent aussi du parcours scolaire à l'université: le type d'établissement fréquenté, le régime d'études et le domaine d'études (tableau 4). Par exemple, les étudiants inscrits à plein temps au début du premier programme ont plus de chances de persévérer jusqu'à l'obtention du diplôme (57 \%) que ceux qui ont commencé leur programme d'études à temps partiel (47\%). De même, ces chances sont relativement plus élevées chez ceux qui s'orientent dans les domaines des sciences et techniques de la vie $(72 \%)$ et des sciences pures et des techniques physiques (68\%) que ceux qui optent pour les sciences humaines (53\%).

Tableau 4

Pourcentage d'obtention du diplôme selon les caractéristiques de la scolarité universitaire (\%)

\begin{tabular}{ccc}
\hline & Sans diplôme & Diplôme \\
\hline Type d'établissement universitaire $\left(\chi^{2}(1)=9,90 ; \mathrm{NS}\right)$ & 44 & 56 \\
Ancienne université & 50 & 50 \\
Nouvelle université & & 57 \\
Régime d'études au premier programme $\left(\chi^{2}(1)=28,62 ; \mathrm{p}<0,001\right)$ & 43 & 47 \\
Temps plein & 53 & 72 \\
Temps partiel & & 68 \\
Champ d'études $\left(\chi^{2}(4)=41,33 ; p<0,01\right)$ & 28 & 64 \\
Sciences et techniques de la vie & 32 & 53 \\
Sciences pures et techniques physiques & 36 & 63 \\
Sciences sociales et administration & 47 & 56 \\
Sciences humaines & 37 & 44 \\
Arts et lettres & Ensemble des répondants & \\
\end{tabular}

NS : non significatif à $\mathrm{p} \geq=0,05$.

Afin d'examiner l'influence relative de chacune des variables indépendantes, une analyse multivariée a été effectuée. Le modèle d'analyse comprend seulement les variables pour lesquelles l'analyse bivariée a révélé une influence significative sur la probabilité d'obtenir le diplôme de baccalauréat. Comme l'indique le tableau 2, il n'existe pas de différence significative entre le taux d'obtention du diplôme des ÉPG et celui des NÉPG. Toutefois, cela ne veut pas dire qu'il ne puisse pas y avoir de différences quant aux facteurs influençant la persévérance des ÉPG et celle des NÉPG. Dans cette optique, des analyses séparées sont effectuées pour les ÉPG et les deux autres groupes afın d'examiner si l'influence de ces variables diffère selon le capital scolaire des parents.

Les résultats (tableau 5) montrent que les variables suivantes, liées au parcours scolaire, exercent une influence significative sur les chances d'obtenir le diplôme de baccalauréat : l'année d'entrée à l'université, la moyenne générale au secondaire, le niveau d'aspiration scolaire, le temps consacré aux devoirs durant les études secondaires et le fait d'avoir interrompu les études. 
Tableau 5

Influence des caractéristiques d'appartenance sociale et culturelle et du parcours scolaire sur l'obtention du diplôme de baccalauréat au Canada - Rapports des cotes de régression logistique

\begin{tabular}{|c|c|c|c|}
\hline & ÉPG & NÉPG collégial & $\begin{array}{c}\text { NÉPG } \\
\text { universitaire }\end{array}$ \\
\hline \multicolumn{4}{|l|}{ Occupation du père } \\
\hline Cadre supérieur ou directeur & 0,89 & 1,46 & 1,01 \\
\hline Poste professionnel & 0,39 & 0,92 & 1,22 \\
\hline Poste technique ou paraprofessionnel & Réf. & Réf & Réf. \\
\hline Poste intermédiaire & 0,81 & $0,48^{*}$ & 1,22 \\
\hline Poste élémentaire et de manœuvre & 1,02 & $0,31 *$ & $1,91 * *$ \\
\hline Sans emploi ou retraité & 0,11 & $0,14 *$ & 0,46 \\
\hline \multicolumn{4}{|l|}{ Sexe } \\
\hline Femme & 1,39 & 1,28 & $1,48^{*}$ \\
\hline Homme & Réf. & Réf. & Réf. \\
\hline \multicolumn{4}{|l|}{ Province de résidence } \\
\hline Terre-Neuve-et-Labrador & 0,59 & $0,17 * * *$ & $0,24 * *$ \\
\hline Île-du-Prince-Édouard & 0,42 & 1,29 & 1,52 \\
\hline Nouvelle-Écosse & 0,54 & 0,40 & 1,71 \\
\hline Nouveau-Brunswick & 0,56 & 2,39 & ,76 \\
\hline Québec & 0,80 & 1,39 & 1,12 \\
\hline Manitoba & 0,64 & 0,58 & 0,94 \\
\hline Saskatchewan & $0,45^{*}$ & 0,50 & $0,53 *$ \\
\hline Alberta & 0,40 & 1,10 & 0,62 \\
\hline Colombie-Britannique & 1,25 & 0,42 & 0,75 \\
\hline Ontario & Réf. & Réf. & Réf. \\
\hline \multicolumn{4}{|l|}{ Moyenne générale } \\
\hline $90-100 \%$ & $18,29^{* * * *}$ & 3,87 & 2,54 \\
\hline $80-89 \%$ & $8,66^{* * *}$ & 2,50 & 2,64 \\
\hline $70-79 \%$ & $9,03^{* * *}$ & 1,01 & 1,30 \\
\hline Moins de $70 \%$ & Réf. & Réf. & Réf. \\
\hline \multicolumn{4}{|l|}{ Temps consacré aux devoirs par semaine } \\
\hline 8 heures ou plus & 2,57 & $3,03^{*}$ & $5,50^{*}$ \\
\hline 4 à 7 heures & 2,02 & 2,14 & $4,95^{*}$ \\
\hline 1 à 3 heure(s) & 2,44 & 1,03 & $5,47^{*}$ \\
\hline Moins d'une heure & Réf. & Réf. & Réf. \\
\hline \multicolumn{4}{|l|}{ Avoir connu un décrochage scolaire } \\
\hline Oui & 0,08 & $0,003^{* * *}$ & 0,66 \\
\hline Non & Réf. & Réf. & Réf. \\
\hline \multicolumn{4}{|l|}{ Interruption d'études avant l'université } \\
\hline Oui & $0,08^{* * * *}$ & 0,52 & 0,44 \\
\hline Non & Réf. & Réf. & Réf. \\
\hline \multicolumn{4}{|l|}{ Niveau d'aspirations scolaires } \\
\hline Études de deuxième cycle universitaire & $3,53^{*}$ & 2,47 & 1,70 \\
\hline Études de premier cycle universitaire & $3,02 *$ & $2,43^{*}$ & 1,37 \\
\hline Études collégiales & Réf. & Réf. & Réf. \\
\hline \multicolumn{4}{|l|}{ Régime d'études } \\
\hline Temps partiel & 0,81 & 1,01 & $0,58 *$ \\
\hline Temps plein & Réf. & Réf. & Réf. \\
\hline \multicolumn{4}{|l|}{ Champ d'études } \\
\hline Sciences et techniques de la vie & 0,93 & $1,82 *$ & 1,01 \\
\hline
\end{tabular}




\begin{tabular}{|c|c|c|c|c|}
\hline \multicolumn{2}{|c|}{ Sciences pures et techniques physiques } & 0,81 & 0,66 & 0,93 \\
\hline \multicolumn{2}{|c|}{ Sciences humaines } & 0,45 & 0,51 & $0,52 *$ \\
\hline \multicolumn{2}{|l|}{ Arts et lettres } & 0,48 & 0,67 & 1,33 \\
\hline \multicolumn{2}{|c|}{ Sciences sociales et administration } & Réf. & Réf. & Réf. \\
\hline \multicolumn{5}{|c|}{ Année d'entrée à l’université } \\
\hline \multicolumn{2}{|l|}{$1997-1998$} & $3,50^{* *}$ & $8,82^{* * *}$ & $6,65^{* * *}$ \\
\hline \multicolumn{2}{|l|}{$1999-2000$} & 0,95 & $2,31^{*}$ & $2,65^{* * *}$ \\
\hline \multirow[t]{5}{*}{ Après 2000} & & Réf. & Réf. & Réf. \\
\hline & Pseudo-R ${ }^{2}$ & 0,23 & 0,23 & 0,18 \\
\hline & $\chi_{\text {de Wald }}^{2}$ & $110,49 * * *$ & $114,19 * * *$ & $145,86^{* * *}$ \\
\hline & $\mathrm{dl}$ & 32 & 32 & 32 \\
\hline & $\mathrm{N}$ & 809 & 788 & 1346 \\
\hline
\end{tabular}

${ }^{*} \mathrm{p}<0,05 ;{ }^{* *} \mathrm{p}<0,01 ;{ }^{* * * *} \mathrm{p}<0,001$

À l'exception de l'année d'entrée aux études postsecondaires, l'influence des autres variables est fonction du statut d'ÉPG ou de NÉPG. D'une manière générale, les ÉPG qui obtiennent le diplôme de baccalauréat se distinguent de ceux qui ne l'obtiennent pas par une moyenne générale élevée au secondaire, un parcours scolaire régulier (sans aucune interruption d'études) et un niveau d'aspirations scolaires élevé. Pour les NÉPG collégiaux, les chances d'obtenir un diplôme de baccalauréat sont plus élevées chez les personnes affichant un niveau élevé d'aspirations scolaires, mais faibles chez ceux qui ont vécu une expérience d'interruption temporaire des études. Par ailleurs, les chances d'obtenir un diplôme de baccalauréat sont plus faibles chez les NÉPG collégiaux dont les pères exercent des emplois associés à des postes intermédiaires, élémentaires et de manœuvres ou sont à la retraite. Chez les NÉPG universitaires, ces chances ont tendance à s'accroître chez les femmes, chez ceux qui avaient l'habitude de consacrer beaucoup plus de temps aux devoirs et chez ceux dont les pères exercent un emploi de niveau élémentaire ou de manœuvre. Il y a donc lieu de constater, dans le cas des NÉPG, l'effet persistant résiduel de l'origine sociale sur l'obtention du diplôme qui s'exerce via l'occupation du père.

Quel que soit le statut d'ÉPG, la date d'entrée joue un rôle déterminant : la probabilité d'obtenir le diplôme de baccalauréat est plus élevée chez ceux qui commencent les études universitaires plus tôt. Elle est aussi nettement plus élevée chez ceux qui sont entrés à l'université durant la période 1997-1998.

Le tableau 6 montre que les étudiants dont les notes et le niveau d'aspiration scolaire étaient élevés au secondaire ont entrepris des études universitaires plus tôt que ceux dont les notes ou les aspirations scolaires étaient plus faibles. Ainsi, parmi les étudiants dont la moyenne variait entre 90 et $100 \%, 90 \%$ sont entrés à l'université entre 1997 et 2000, soit $40 \%$ au cours de la période 1997-1998 et $50 \%$ au cours de la période 1999-2000. Par contre, parmi les étudiants qui présentaient une moyenne inférieure à $70 \%$, seulement $47 \%$ étaient à l'université entre 1997 et 2000, soit $12 \%$ en 1997-1998 et $35 \%$ en 1999-2000. À l'inverse, seulement $10 \%$ des étudiants dont la moyenne se situait entre 90 et $100 \%$ ont pris du retard pour entrer à l'université après 2000, alors que cette proportion est de $53 \%$ chez ceux dont la moyenne se situait en deçà de $70 \%$. 
Tableau 6

Pourcentage des répondants selon la moyenne générale au secondaire, les aspirations scolaires et l'année d'entrée à l'université

\begin{tabular}{cccc}
\hline & $1997-1998$ & $1999-2000$ & 2001 ou après \\
\hline Moyenne générale $\left(\chi^{2}(6)=370,89 ; p<0,001\right)$ & 40 & 50 & 10 \\
$90-100 \%$ & 32 & 50 & 18 \\
$80-89 \%$ & 22 & 44 & 33 \\
$70-79 \%$ & 12 & 35 & 53 \\
Moins de $70 \%$ & & \\
Niveau d'aspirations scolaires au secondaire $\left(\chi^{2}(4)=595,20 ; p<0,001\right)$ & 52 & 13 \\
Études de deuxième cycle universitaire & 35 & 51 & 22 \\
Études de premier cycle universitaire & 27 & 19 & 62 \\
Études collégiales & 19 & & \\
\hline
\end{tabular}

En ce qui touche ceux qui aspiraient à un diplôme d'études universitaires de deuxième cycle, $87 \%$ sont entrés à l'université entre 1997 et 2000, alors que seulement $38 \%$ de ceux qui envisageaient un diplôme d'études collégiales sont entrés à la même période.

\section{Pistes interprétatives}

Au Canada, l'accès à l'enseignement postsecondaire est influencé par diverses dimensions. Plus particulièrement, les facteurs caractérisant la scolarité au secondaire ont une influence forte sur l'accès à l'université, ce qui reflète le fonctionnement méritocratique du système scolaire et de l'université. Il existe donc des mécanismes de sélection sur la base des résultats scolaires et de la nature du parcours au secondaire. Fautil pour autant se réjouir de ces résultats qui soulignent aussi une indépendance des effets de la scolarité antérieure par rapport à l'origine sociale? Deux raisons nous poussent à relativiser cette conclusion. D'une part, les effets du décrochage scolaire se font toujours sentir en réduisant la probabilité d'accéder aux études universitaires. Le dossier scolaire conserve les traces d'une scolarité caractérisée par des difficultés : les individus qui ont décroché ne désirent pas aller à l'université, indépendamment de leurs notes. D'autre part, la sélection n'est pas uniquement scolaire; elle est également sociale. En effet, l'accès des ÉPG aux études universitaires est plus faible que celui des NÉPG. Ainsi, les jeunes dont les parents ont fait des études postsecondaires, notamment universitaires, participent davantage aux études universitaires que les ÉPG. En plus, nous avons constaté que la catégorie socioprofessionnelle du père influence l'accès. Ainsi, l'analyse permet de constater l'effet de l'origine sociale sur la scolarisation, non seulement par le capital culturel au sens large du terme, mais aussi par le capital scolaire. En d'autres mots, la " démocratisation " des études universitaires est ségrégative, pour reprendre l'expression de Merle (2000), et elle est marquée du sceau de la reproduction sociale.

$\mathrm{Au}$ Canada, l'éducation relève de la juridiction des provinces et, historiquement, chacune d'entre elles a créé et aménagé son système d'éducation. Les différences des systèmes entre les provinces se font sentir quant à la probabilité d'accéder à l'université, laissant penser que l'organisation de l'offre des études universitaires a un effet sur l'accès. L'analyse permet d'identifier les provinces (Québec, Alberta, ColombieBritannique) où les taux d'accès aux études universitaires sont comparables à celui de l'Ontario et celles où ces taux d'accès sont significativement plus élevés que celui de 
l'Ontario (les provinces maritimes, le Manitoba et la Saskatchewan). Ce phénomène pourrait s'expliquer par l'importance des établissements universitaires au sein de la structure de l'enseignement postsecondaire de chacune de ces provinces. Celles où les collèges sont moins développés offriraient un accès à l'université plus élevé par rapport à la province de référence où les collèges communautaires sont bien implantés.

Qu'arrive-t-il aux étudiants une fois intégrés à la formation universitaire? Les ÉPG et les NÉPG ont des chances égales d'obtenir le diplôme de baccalauréat, ce qui contraste avec la situation des ÉPG étatsuniens. Doit-on y voir un effet de la méritocratie scolaire et considérer que les ÉPG canadiens qui poursuivent des études universitaires sont " semblables " aux NÉPG, ce qui laisse supposer que les facteurs qui influencent la persévérance des ÉPG relèvent principalement de la scolarité antérieure? Les résultats obtenus appuient cette hypothèse. Les ÉPG poursuivant des études universitaires ressemblent fortement aux autres étudiants quant au rendement scolaire et aux caractéristiques du parcours scolaire, notamment en ce qui concerne les moyennes du secondaire et le fait d'avoir connu ou non une interruption d'études. Dans la mesure où les ÉPG accèdent à l'université dans une proportion nettement plus faible que les NÉPG, on peut soutenir l'idée que les ÉPG qui poursuivent des études universitaires y arrivent au terme d'un processus de sur-sélection. Il s'agirait d'élèves ayant des compétences scolaires relativement élevées. Or, comme l'a noté Duru-Bellat (2002) pour les élèves performants, la poursuite des études universitaires est un acte normal, voire évident, peu importe leur origine sociale. Par contre, à compétences faibles comparables, les élèves d'origine sociale défavorisée aspirent moins aux études universitaires, car ils préfèrent ne pas s'aventurer dans un projet pour lequel la probabilité d'un échec leur semble trop élevée. Tout compte fait, les parcours scolaires s'inscrivent dans un processus d'auto-sélection.

Un dernier résultat met aussi en évidence une autre logique présente dans le fonctionnement du système éducatif, celle de la mobilisation scolaire de certains groupes sociaux - par exemple, les femmes et les immigrants - pour qui les études postsecondaires, et les études universitaires en particulier, représentent un instrument d'intégration socioéconomique, voire de mobilité sociale. Cet aspect n'est pas nouveau, les résultats de recherche soulignent depuis longtemps les variations de scolarisation selon l'appartenance ethnoculturelle.

Dans la mesure où les ÉPG viennent, pour la plupart, de familles plus défavorisées sur les plans économiques et scolaires, leur faible participation aux études universitaires tient à la fois à leurs compétences scolaires et à leurs aspirations moins élevées à la fin des études secondaires. Cela expliquerait d'ailleurs l'existence d'une relation fortement significative entre la note moyenne obtenue à la fin du secondaire et la persévérance à l'université chez les ÉPG, alors que cette même relation ne se retrouve pas chez les NÉPG. Comme ils bénéficient de peu ou de moins de ressources de la part de leurs familles, les ÉPG qui accèdent aux études universitaires se caractérisent particulièrement par de meilleures habiletés à composer avec leur métier d'étudiant et par un niveau plus élevé d'engagement dans les études.

Enfin, on constate entre les provinces, voire entre les universités, des différences qui pourraient faire l'objet d'une exploration plus poussée, ne serait-ce qu'en tenant compte, à la fois, des missions dont se réclament les établissements, et de l'organisation des services fournis aux étudiants. 


\section{CONCLUSION}

Le concept d'étudiant de première génération a suscité au cours des dernières années un intérêt croissant chez les chercheurs des États-Unis, mais il a connu peu d'écho dans d'autres sociétés. Dans cet article, nous avons cherché à opérationnaliser le " capital scolaire familial " en appliquant ce concept au contexte canadien. En ce sens, nous avons procédé à une importation conceptuelle. Les résultats sont en partie probants, confirmant le poids de l'origine sociale et culturelle dans l'accès aux études universitaires, mais pas dans l'obtention du baccalauréat au Canada. Cette analyse mérite d'être poursuivie par une comparaison systémique entre pays afın de mieux évaluer le poids des trois logiques sociales à l'œuvre en éducation, à savoir : la méritocratie scolaire, la reproduction sociale et la mobilisation pour les études.

Le concept d'ÉPG présente aussi l'avantage d'être simple et facile à opérationnaliser. À l'heure des classements et de la production de multiples indicateurs visant à mieux comprendre le développement du système éducatif, cette notion peut devenir un indicateur pertinent pour mesurer le degré de démocratisation des institutions d'enseignement postsecondaire.

\section{BIBLIOGRAPHIE}

Adachi, F. F. (1979). Analysis of the first generation college student population. A new concept in higher education. Laramie: University of Wyoming (texte non publié).

Auclair, R., Bélanger, P., Doray, P., Gallien, M., Groleau, A., Mason, L. \& Mercier, P. (2008). Les étudiants de première génération : un concept prometteur? Transitions - Note 2. Montréal : Fondation canadienne des bourses d'études du millénaire http:// www.cirst.uqam.ca/Portals/0/docs/projet_transitions/Note\%202.pdf (Consulté le 23 septembre 2010).

Billson, J. M. \& Brooks-Terry, M. (1982). In search of the silken purse: factors in attrition among first-generation students. College and University, 58(1), 57-75.

Brown, H. E. \& Burkhardt, R. L. (1999, May 30-June 3). Predicting student success: the relative impact of ethnicity, income, and parental education. Document présenté lors du forum annuel de l'Association for Institutional Research, Seattle, WA.

Chen, X. \& Carroll, D. C. (2005). First generation students in postsecondary education: A look at their college transcripts. Washington, D.C. : U.S. Department of Education, National Center for Education Statistics.

Choy, S. (2001). Students whose parents did not go to college: postsecondary access, persistence and attainment. Washington, D.C. : U.S. Department of Education, National Center for Education Statistics.

Dennis, J. M., Phinney, J. S. \& Chuateco, L.I. (2005). The role of motivation, parental support, and peer support in the academic success of ethnic minority first-generation college students. Journal of College Student Development, 46(3), 223-236.

Dubet, F. (1994). Dimensions et figures de l'expérience étudiante dans l'université de masse. Revue française de sociologie, 35(4), 511-532. 
Duggan, M. B. (2002). The effect of social capital on the first-year persistence of first generation college students. Boston : University of Massachusetts (Doctorat Éducation).

Esping-Andersen, G. (1999). Les trois mondes de l'État providence : essai sur le capitalisme moderne. Paris : Presses universitaires de France.

Finnie, R., Laporte, C., \& Lascelles, E. (2004). Antécédents familiaux et accès aux études postsecondaires : que s'est-il passé pendant les années 1990? Document de recherche sur les études analytiques, Catalogue 11F0019MIF2004. Ottawa : Statistique Canada.

Finnie, R., Lascelles, E., \& Sweetman, A. (2005). Qui poursuit des études supérieures? L'incidence directe et indirecte des antécédents familiaux sur l'accès aux études postsecondaires. Document de recherche sur les études analytiques, Catalogue 11F0019MIF2005237. Ottawa : Statistique Canada.

Frenette, M. (2003). Accès au collège et à l'université : est-ce que la distance importe? Document de recherche sur les études analytiques, Catalogue 11F0019MIF2003201. Ottawa : Statistique Canada.

Frenette, M. (2002). Trop loin pour continuer? Distance par rapport à l établissement et inscription à l université. Document de recherche sur les études analytiques, Catalogue 11F0019MIF2002191. Ottawa : Statistique Canada.

Grayson, J. P. (1997). Academic achievement of first-generation students in a canadian university. Research in Higher Education, 38(6), 659-676.

Hahs-Vaughn, D. (2004). The impact of parents' education level on college students: An analysis using the beginning post-secondary students longitudinal study 1990-92/94. Journal of College Student Development, 45(5), 483-500.

Horn L., \& Nuñez, A.-M. (2000). Mapping the road to college: first-generation students' math track, planning strategies, and context of support. Washington, D.C. : U.S. Department of Education, National Center for Education Statistics. http://nces. ed.gov/pubs2000/2000153.pdf (Consulté le 23 septembre 2010).

Inman, W. E. \& Mayes, L. (1999). The importance of being first: Unique characteristics of first generation community college students. Community College Review, 26(4), 3-22.

Ishitani, T. T. (2003). A longitudinal approach to assessing attrition behavior among first-generation students: Time-varying effects of pre-college characteristics. Research in Higher Education, 44(4), 433-449.

Ishitani, T. T. (2006). Studying attrition and degree completion behaviour among first-generation college students in the United States. The Journal of Higher Education, 77(5), 861-885.

Kamanzi, C., Doray, P., Murdoch, J., Moulin, S., Comoe, É., Groleau, A., Leroy, C., \& Dufresne, F. (2009). L'influence des déterminants sociaux et culturels sur les parcours et transition dans l'enseignement supérieur. Note 6 - Projet Transitions. Montréal : Fondation canadienne des bourses d'études du millénaire. 
Lara, J. (1992). Reflections: Bridging cultures. New Directions for Community Colleges, (80), 65-70.

Lehmann, W. (2007). "I just didn't feel like I fit in": The role of habitus in university dropout decisions. Canadian Journal of Higher Education, 37(2), 89-110.

Lohfink, M. M. \& Paulsen, M. B. (2005). Comparing the determinants of persistence for first-generation and continuing-generation students. Journal of College Student Development. 46(4), 409-428.

London, H. B. (1989). Breaking away: a study of first-generation college students and their families. American Journal of Education, 97(1), 144-170.

London, H. B. (1996). How college affects first-generation students. About campus, 1(5), 9-23.

Merle, P. (2000). Le concept de démocratisation de l'institution scolaire : une typologie et sa mise à l'épreuve. Population, 55(1), 15-50.

Naumann, W. C., Bandalos, D., \& Gutkins, T. B. (2003). Identifying variables that predict college success for first-generation college students. The Journal of College Admission, (181), 4-9.

Nuñez, A.-M., Cuccaro-Alamin, S. \& Carroll, C.D. (1998). First-generation students: undergraduates whose parents never enrolled in postsecondary education. Washington, D.C: U.S. Department of Education, National Center for Education Statistics.

OCDE (2005). Regards sur l'éducation. Paris : OCDE.

Pascarella, E.T., Wolniak, G. C., Pierson, C. T., \& Terenzini P. T. (2003). Experiences and outcomes of first-generation students in community colleges. Journal of College Student Development, 44(3), 420-429.

Pascarella, E.T., Wolniak, G. C., Pierson, C. T., \& Terenzini, P. T. (2004). Firstgeneration college students: additional evidence on college experiences and outcomes. Journal of Higher Education, 75(3), 249-284.

Penrose, A. M. (2002). Academic literacy perceptions and performances: comparing first-generation and continuing-generation students. Research in the Teaching of English, 36, 437-461.

Pike, G. R. \& Kuh, G. D. (2005). First- and second-generation college students: A comparison of their engagement and intellectual development. Journal of Higher Education, 76(3), 276-300.

Rahman, A., Situ J., \& Jimmo V. (2005). Participation aux études postsecondaires : Résultats de l'Enquête sur la dynamique du travail et du revenu. Document de recherche sur les études analytiques, Catalogue 81-595-MIF2005036. Ottawa : Statistique Canada.

Rendon, L. I. (1992). From the barrio to the academy: Revelations of a Mexican American "scholarship girl". New Directions for Community Colleges, (80) 55-64.

Rodriguez, R. (1975). Going home again: The new American scholarship boy. American Scholar, 44(1), 15-28. 
Rodriguez, R. (1982). Hunger of memory: The education of Richard Rodriguez - an autobiography. Boston : Godine.

Saint-Arnaud, S. \& Bernard, P. (2003). Convergence ou résilience? Une analyse de classification hiérarchique des régimes providentiels. Sociologie et sociétés, $X X X V(1)$, 65-93.

Shaienks, D. \& Gluszynski, T. (2007). Participation aux études postsecondaires : diplômés, persévérants et décrocheurs, résultats de l'EJET, 4e cycle. Ottawa : Statistique Canada/Ressources humaines et développement des compétences Canada, Division de la culture, tourisme et centre de la statistique de l'éducation.

Statistique Canada (2005). Effectifs universitaires, 2002-2004. Le Quotidien, 11 octobre 2005, Catalogue 11-001-XIF, 5-10.

Terenzini, P.T., Springer, L., Yaeger, P.M., Pascarella, E.T., \& Nora, A. (1996). Firstgeneration college students: Characteristics, experiences, and cognitive development. Research in Higher Education, 37(1), 1-22.

Warburton, E. C., Bugarin, R., \& Nuñez, A-M. (2001). Bridging the gap: academic preparation and postsecondary success of first-generation students. Washington, D.C. : U.S. Department of Education, National Center for Education Statistics.

York-Anderson, D. C. \& Bowman, S.L. (1991). Assessing the college knowledge of first-generation and second-generation college students. Journal of College Student Development. 32(2), 116-122.

\section{CONTACT INFORMATION}

Pierre Canisius Kamanzi

Centre interuniversitaire de recherche sur la science et la technologie

Université du Québec à Montréal

405 Sainte-Catherine St E

Montreal, Canada H2L 2C4, Canada

Email: kamanzi.pierre_canisius@uqam.ca

Pierre Canisius Kamanzi is a researcher at the Interuniversity Research Center on Science and Technology (CIRST, UQAM). His research focuses on academic trajectories (access to and pursuit of post-university study) and entrance into the work force.

Pierre Doray est professeur titulaire au département de sociologie de l'Université du Québec à Montréal. Il est membre régulier du Centre interuniversitaire de recherche sur la science et la technologie (CIRST), qu'il a dirigé de 2005-2009. Il est l'auteur de nombreuses publications et communications scientifiques portant sur les parcours étudiants, le développement de la formation des adultes ainsi que la formation professionnelle et technique.

Sylvie Bonin est agente de recherche à la Direction de l'analyse et de la recherche institutionnelle (DARI) de l'Université du Québec depuis 2002. Sa formation en mathématiques et en économétrie et son expertise au niveau de l'analyse des données l'ont 
amené à intégrer l'équipe de la DARI où elle collabore aux divers projets concernant les étudiants, notamment l'analyse des données d'enquêtes, le suivi des cohortes étudiantes et la production des statistiques officielles.

Amélie Groleau est doctorante en sociologie à l'Université du Québec à Montréal et à l'École des hautes études en sciences sociales à Paris. Ses intérêts de recherche portent sur le processus de transmission intergénérationnelle, l'enseignement supérieur, les parcours scolaires et les scolarités atypiques. Au plan professionnel, Mlle Groleau a collaboré pendant plus de deux ans au projet Transitions, sur les parcours scolaires dans l'enseignement postsecondaire au Québec et au Canada.

Jake Murdoch is associate professor at the department of administration and educational foundations of the University of Montreal. His research interests cover notably the comparison of education systems (across Canada and abroad) and the study of educational pathways (particularly those of postsecondary students). His is member of various pan-canadian and international networks on higher education (Canadian Society for the Study of Higher Education and the Consortium of Higher Education Researchers), and has taken part in large-scale comparative studies on higher education.

\section{NOTES}

1. La réalisation de ce texte a été rendue possible grâce au soutien de la Fondation canadienne des bourses du millénaire (FCBM) et du Conseil de recherche en sciences humaines du Canada (CRSH). Les auteurs tiennent aussi à remercier les évaluateurs anonymes pour leurs commentaires, combien pertinents. 